\title{
Application of new modifying models and lifestyle changes and its role in providing better treatment for diabetes mellitus
}

\author{
Khaled M. Hassan ${ }^{1 *}$, Moutaz A. Alghamdi², Wesam E. Althagafi ${ }^{3}$, Salem M. Alqarni ${ }^{4}$, \\ Ammar K. Al Ramadhan ${ }^{5}$, Ruqiah M. Al Darwish ${ }^{5}$, Rakan R. Alanazi ${ }^{5}$, Basim H. Alshehri ${ }^{4}$, \\ Abdulraheem A. Albalawi ${ }^{6}$, Muqdad A. Alqaisoom ${ }^{5}$, Abdullah M. Hussain ${ }^{7}$, \\ Sumyyah U. Almezher ${ }^{4}$, Hadeer A. Rajab ${ }^{6}$, Ramah H. Ghazi ${ }^{7}$, Haneen A. Al Asiri ${ }^{4}$, \\ Ali A. Aljallal ${ }^{8}$
}

\author{
${ }^{1}$ Department of Medicine, Consultant Family Medicine, Saudi Arabia \\ ${ }^{2}$ Department of Medicine, AlFarabi College, Saudi Arabia \\ ${ }^{3}$ Department of Medicine, King Saud Bin Abdulaziz University for Health Sciences, Saudi Arabia \\ ${ }^{4}$ Department of Medicine, King Khalid University, Saudi Arabia \\ ${ }^{5}$ Department of Medicine, Imam Abdulrahman Bin Faisal University, Saudi Arabia \\ ${ }^{6}$ Department of Medicine, King Abdulaziz University, Saudi Arabia \\ ${ }^{7}$ Department of Medicine, Umm Alqurra University, Saudi Arabia \\ ${ }^{8}$ Department of Medicine, Imam Muhammad Bin Saud University, Saudi Arabia
}

Received: 29 August 2020

Revised: 12 September 2020

Accepted: 14 September 2020

\section{*Correspondence:}

Dr. Khaled M. Hassan,

E-mail: Khaled-h-8@outlook.sa

Copyright: ( $)$ the author(s), publisher and licensee Medip Academy. This is an open-access article distributed under the terms of the Creative Commons Attribution Non-Commercial License, which permits unrestricted non-commercial use, distribution, and reproduction in any medium, provided the original work is properly cited.

\begin{abstract}
Diabetes is one of the main threats to human health in this century. The drastic increase in incidence of diabetes worldwide has been attributed to distinct changes in human behaviour and lifestyle during the last century. To prevent the personal and socio-economic burden of diabetes effort to prevent the disease needs to start before the onset of diabetes and address all susceptibility factors. Four recent studies have shown that prevention of type 2, diabetes is possible and that reinforced lifestyle interventions or modification can significantly reduce the onset of the disease. The studies repeatedly showed that for about $60 \%$ of the persons with increased diabetes risk, compared to a control group, the diabetes was prevented from developing. Early pharmacologic preventive strategies have yielded 25-30\%. These studies have convincingly demonstrated that the primary aim in prevention of type 2 diabetes is the stabilization of glucose tolerance due to improvement of insulin resistance. Based on the studies intervention protocol focuses on achieving 5 core goals. With respect to the world wide burden of diabetes this studies offer a compelling evidence base for the important translation of the research findings into community-based prevention strategies a development of a National diabetes prevention program. The workgroup "diabetes prevention" at the German diabetes association together with the diabetes foundation developed a concept for a National program. To realize this, a large number of partners are necessary. For the population based implementation of such a program the intervention is primarily based on reinforced behaviour modification (lifestyle and physical activity). As add on the pharmaco prevention of type 2 diabetes will be from increasing importance.
\end{abstract}

Keywords: New modifying models, Life style change, Providing better treatment, Diabetes mellitus 


\section{INTRODUCTION}

Diabetes is a metabolic disorder characterized by a chronic hyperglycemia (too high blood sugar levels) linked to deficiency, either in insulin secretion, or in the action of insulin, or of both. ${ }^{1}$ Eventually carrier of micro and macro vascular complications severe and disabling in certain target organs such as the heart (heart failure, myocardial infarction), the central nervous system (stroke) and peripheral (neuropathy), the kidney(chronic kidney disease, kidney failure), eye (retinopathy) and foot(foot ulceration, diabetic foot). ${ }^{2}$ It is a major health problem public by its significant and increasing prevalence on the one hand, and its socio-economic impact on the other hand. ${ }^{3}$ As a result, diabetes constitutes currently one of the most worrying pathologies in both countries industrialized than in developing countries. In the world,

World Health Organization (WHO) talks about global epidemic (all types of diabetes combined). In 2014 the global prevalence was estimated at $9 \%$ in adults over 18 years. In 2012, diabetes was responsible for 1.5 million deaths worldwide and type 2 represented just over $90 \%$ of cases and $80 \%$ of these deaths occur in countries low or middle income. ${ }^{4}$

\section{Lifestyle prevention of type 2 diabetes}

T2DM is predestined for effective primary prevention, since a large part of the disease is caused by lifestyle factors and these can be influenced by appropriate interventions (organization, 1994 \#2906). However, the challenge is to identify people who are at an increased risk of the disease in the diagnosis free interval or earlier. It is important to determine which lifestyle or drug interventions will be most effective in preventing or delaying diabetes.

Currently, in Morocco, a country in the midst of a transition phase demographic, nutritional and epidemiological, diabetes is on its way as an important public health issue and represents a challenge to which doctors are confronted in their daily practice. ${ }^{5}$ The studies showed figures to be around $6.6 \%$, or over a million and half Moroccans in 2010; this figure will reach 2.5 million on the horizon $2030 .^{6}$

We know that physical activity regular lowers blood sugar, improves insulin sensitivity, decreases fat mass, improves the lipid balance and, in general, helps control risk factors and prevent complications from DT2. Its effects on quality of life, on self-esteem and restoration of social ties are also well established, ${ }^{7}$ an increase in physical activity to 30 minutes per day and a weight reduction of approximately $5 \% .^{8,9}$

\section{METHODS}

The finding of this study is a compilation of previous research. This exploratory qualitative research, carried out on adults with diabetes type 2 insulin treated, allows to describe according to their perceptions, the factors that influence their diabetes self-management. ${ }^{9}$

\section{RESULTS}

The results of several large international studies provide initial answers and provide high evidence that the prevention of diabetes mellitus with lifestyle intervention or the early use of medication is possible and effective in people at risk. ${ }^{10-14,15}$ In the studies mentioned, it was repeatedly shown that lifestyle intervention in the early stages of the disease could successfully prevent or delay the onset of diabetes for almost $60 \%$ of those affected, and early drug intervention for about a quarter of those at risk.

In the Chinese Da Quing diabetes prevention study, the effect of a "lifestyle modification" on the conversion from the IGT stage to T2DM was examined. The study included 577 people with impaired glucose tolerance who were randomly included in control and intervention groups. ${ }^{13}$ The cumulative incidence of diabetes after 6 years in the control group was $67.7 \%$ (95\% CI, 59.8-75.2) compared to the "lifestyle group" with $41.1 \%$ (95\% CI, 33.4-49.4). For example, through intensive diet training and exercise programs in Chinese patients with impaired glucose tolerance, a diabetes risk reduction of $46 \%$ was achieved. ${ }^{13}$

In the Finnish diabetes prevention study (DPS), in which subjects with impaired glucose tolerance were also examined $(n=522)$, the incidence rate in a three-year follow-up was 22.9 through "lifestyle modification" percent can be reduced to $10.2 \%$, which corresponds to a diabetes risk reduction of $58 \% .^{15-17}$

The test persons in the "lifestyle group" were able to reduce their body weight by $5.6 \mathrm{~kg}$ during the observation period. Subjects treated with metformin had a weight reduction of $2.1 \mathrm{~kg}$ and the control group $0.1 \mathrm{~kg}$. The incidence of diabetes was 4.8 people per 100 patientyears in the lifestyle group compared to 7.8 in the metformin treated group and 11.0 people in the untreated control group. In this study, too, the lifestyle modification proved to be a very effective treatment measure with a "number needed to treat" of 6.9 people. 14 subjects had to be treated with metformin to prevent diabetes. ${ }^{10-16}$

Another pharmacopreventive study, the STOP-NIDDM study, evaluated the effectiveness of acarbose in preventing the occurrence of overt diabetes in people with IGT. ${ }^{12}$ It could be shown that in this study the regular intake of acarbose led to a relative risk reduction of $25 \%$. However, the preventive effect ceased after acarbose was discontinued. ${ }^{12}$ Pre-defined endpoints as secondary outcomes were also reduced under acarbose, namely $49 \%$ fewer cardiovascular events and $34 \%$ fewer new hypertension cases. On the one hand, this indicates the particular importance of correcting postprandial 
hyperglycemia. On the other hand, it is the first evidence that treating IGT not only delays diabetes, but can also significantly reduce cardiovascular risk.

As a result of these studies, target values were formulated, particularly for lifestyle intervention, the implementation of which is crucial for preventing or delaying diabetes. These include, $7 \%$ weight reduction, 150 minutes of physical activity/week, $5 \mathrm{~g}$ fibercontaining fiber $/ 1000 \mathrm{kcal}$ food intake, a maximum of $30 \%$ fat in daily food, a maximum of $10 \%$ saturated fatty acids in the daily diet.

It is interesting here that retrospectively the implementation of one of the target values had almost no preventive effect, but with the continuous implementation of each further target value the effect in the prevention of diabetes increased and with the continuous implementation of four and all five target values over the study period almost $100 \%$ prevention of diabetes was achieved. ${ }^{11}$

The studies prove the possibility of primary diabetes prevention through a successful change in lifestyle, in particular diet and exercise. As a second effect it could be shown convincingly that an early intake of metformin or acarbose can prevent or delay the occurrence of overt diabetes in people with IGT. Especially with regard to the transferability of the effort made in the study to the actual clinical routine, regular pharmacoprevention can represent an important alternative in a future prevention program for a substantial number of people at risk or "non-responders" of lifestyle prevention.

\section{DISCUSSION}

The lifestyle modification included individual nutritional advice appointments and individual advice on physical activity. Compared to the untreated control group, patients with lifestyle modification lost $4.2 \mathrm{~kg}$ in weight, increased their exercise behaviour, lowered blood pressure and improved lipid metabolism. 8 people had to be "treated" to prevent diabetes. Subjects who lost more than $5 \%$ of their body weight had a $70 \%$ reduction in the risk of diabetes compared to people without a corresponding weight reduction. In addition to increased physical activity, weight reduction thus proved to be a decisive effect variable. ${ }^{11-15}$

The aim of the American diabetes primary prevention trial (DPP) was to examine the effect of a "lifestyle modification" and early metformin treatment compared to a placebo group in subjects with IGT on conversion to manifest type 2 diabetes mellitus. ${ }^{10}$ The results show that with a mean observation period of 2.8 years, the risk can be reduced by early metformin treatment by $31 \%$, but by lifestyle modification by $58 \%$ compared to placebo. The management of type 2 diabetic patients must be comprehensive, with the objective of preventing micro and macro vascular complications of disease and ensure a good quality of life. The objective of this study was to study the role of physical activity in the balance of type 2 diabetes.

\section{CONCLUSION}

To meet the challenge of chronic diseases, especially diabetes, studies have shown that the most effective strategies are those based on the quality of the care services offered, but also, and to the same degree on preventive actions targeting behavior, and first of all, therapeutic education and in particular the promotion of the activity physical. The diabetes therapeutic education program, currently in preparation, is an opportunity that must be encouraged, and to ensure all the success factors. As a result, the population-wide implementation of diabetes prevention will be primarily based on a motivation to change lifestyle. The additional effect of a lifestyle change is certainly that, in addition to preventing diabetes through stronger effects on weight, blood pressure and lipid metabolism, the focus is on the prevention of metabolic syndrome. This effect can possibly even outweigh the diabetes preventive effect. Of decisive importance, however, will be how successfully and how stable the people at risk can be motivated to implement the lifestyle change over the long term. Primary prevention of type 2 diabetes mellitus means a lifelong responsibility. To realize such a project, many partners are needed who are involved in this process. The political preparatory work is important here; Scientific and professional organizations are required. But the food and pharmaceutical industries are also key partners. With a convincing evaluation of a national diabetes prevention program, not only will the prevention of diabetes be successfully implemented, but also the prevention of the metabolic syndrome and especially its cardiovascular complications will be possible at the same time. These data are of considerable importance for the practice of diabetology, as they not only conclusively prove the long suspected effectiveness of weight reduction and show the unexpected effectiveness of pharmacotherapy with different active ingredients, but also allow quantitative statements to be made to compare the interventions. Thereafter, weight loss combined with physical activity is the most effective intervention. Furthermore, the early pharmacoprevention of diabetes mellitus is of substantial benefit. In addition to lifestyle changes, early pharmacoprevention of diabetes will be of crucial importance in the future.

\section{Funding: No funding sources Conflict of interest: None declared Ethical approval: Not required}

\section{REFERENCES}

1. Grimaldi A. Treatise on diabetology, 2nd edition. Ed. Medicine Sciences, Flammarion, 2009. 
2. Morel A, Lecoq G, Menninger J. Assessment of the management of diabetes. General Inspectorate Social Affairs. 2012;1:33.

3. Massi-Benedetti M, The Cost of Diabetes Type II in Europe: The CODE-2 Study. Diabetologia. 2002;45:10-5.

4. World Health Organization, diabetes;memory aid. Available at: http://www.who.int/ mediacentre/factsheets /fs $312 / \mathrm{fr} /$. Accessed on 02 May 2015.

5. Farouqil A, Harti M, Nejjari C. Management of diabetes in Morocco: results of the International Diabetes Management Practices Study (IDMPS)Wave 2. Medicine for Metabolic Diseases. 2010;4(6):704-11.

6. Hassoune S, Badri S, Nani S, Belhadi L, Maaroufi A. Assessment of treatment of diabetics by the general practitioner in theKhouribga province (Morocco). EMHJ. 2013;19(1):5-9.

7. Haffner SM. Les sujets prédiabétiques insulinorésistants présentent plus de facteurs de risque athérogènes que les sujets prédiabétiques insulinosensibles: implications pour la prévention des maladies coronariennes pendant l'état prédiabétique. Circulation. 2000;101(9): 975-80.

8. Fontbonne A. Répartition de la graisse corporelle et mortalité par maladie coronarienne chez les sujets présentant une tolérance au glucose altérée ou un diabète sucré: étude prospective de Paris, suivi de 15 ans. Diabetologia. 1992;35(5):464-8.

9. Pal DK. Caractéristiques phénotypiques des crises fébriles familiales: étude cas-témoins. Neurologie. 2003;60(3):410-4.

10. Knowler WC, Barrett-Connor E, Fowler SE, Hamman RF, Lachin JM, Walker EA, et al. Reduction in the incidence of type 2 diabetes with lifestyle intervention or metformin. New England $\mathbf{J}$ Med. 2002;346(6):393-403.

11. Tuomilehto J, Lindström J, Eriksson JG, Valle TT, Hämäläinen $\quad H$, Ilanne-Parikka $P$, et al.
Prevention of type 2 diabetes mellitus by changes in lifestyle among subjects with impaired glucose tolerance. N Engl J Med. 2001;344(18):1343-50.

12. Chiasson JL, Josse RG, Gomis R, Hanefeld M, Karasik A, Laakso M, et al. Acarbose for prevention of type 2 diabetes mellitus: the STOP-NIDDM randomized trial. Lancet, 2002;359:2072-7.

13. Pan XR, Li JW, Hu YH, Wang JX, Yang WY, An $\mathrm{ZX}$, et al. Effects of diet and exercise in preventing NIDDM in people with impaired glucose tolerance. The Da Qing IGT and Diabetes Study. Diabetes Care.1997;20(4):537-44.

14. Lindstrom J, Tuomilehto J. The diabetes risk score: a practical tool to predict type 2 diabetes risk. Diabetes Care. 2003;26(3):725-31.

15. Engelgau MM, Venkat Narayan KM, Vinicor F. Identifying the target population for primary prevention: the trade-offs. Diabetes Care. 2002;25(11):2098-9.

16. Eriksson J, Lindstrom J, Valle T, Aunola S, Hamalainen H, Lakso M, et al. Prevention of Type II diabetes in subjects with impaired glucose tolerance: the Diabetes Prevention Study (DPS) in Finland. Study design and 1-year interim report on the feasibility of the lifestyle intervention program. Diabetologia. 1999;42(7):793-801.

17. Molitch ME, Fujimoto W, Hamman RF, Knowler WC, Diabetes prevention research group. The diabetes prevention program and its global implications. J Am Soc Nephrol. 2003;14:103-7.

Cite this article as: Hassan KM, Alghamdi MA, Althagafi WE, Alqarni SM, Al Ramadhan AK, Darwish RMA, et al. Application of new modifying models and lifestyle changes and its role in providing better treatment for diabetes mellitus. Int $\mathrm{J}$ Community Med Public Health 2020;7:4146-9. 